\title{
Work Hard, Play Hard - A Work Ethic Forged in the Group Oppolzer
}

\author{
Alan C. Spivey*
}

\begin{abstract}
The author gives an account of his work as post-doc at the University of Geneva in the group of W. Oppolzer, putting emphasis on scientific rigour and ingenuity.
\end{abstract}

Keywords: Department of organic chemistry - Enantioselective synthesis · Hydroxyamination

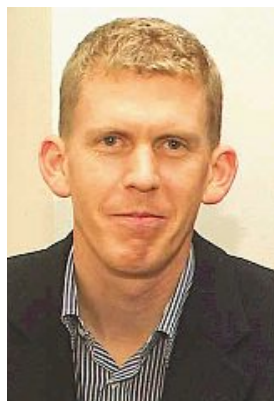

I arrived in Geneva on the evening of Friday 3rd January, 1992. My letter of invitation from Wolfgang Oppolzer stipulated a start date of the 6th Jan and, having some prior knowledge of the temperament of my future supervisor, albeit entirely second-hand in the form of quasi-apocryphal accounts of an individual with high expectations who demanded research precision, I was keen to get settled in and do some reactions as soon as possible. I travelled across town from the airport by tram and on foot; the Département de Chimie Organique was essentially deserted when I made my way to his office on the 1 st floor with my ruc-sac. I was greeted very warmly by the man himself who immediately ushered me into his office and began to discuss potential projects - shuffling through various hand-written notes jammed into a slim filing cabinet drawer and talking fast in English interspersed with German. He rapidly came to the conclusion that I should explore the diastereoselective synthesis of 1,2-amino alcohols by hydroxyamination of the enolate of propiophenone with 1,1-chloronitrosocyclohexane followed by carbonyl/N-O bond reduction (Scheme 1a).

${ }^{*}$ Correspondence: Prof. A. C. Spivey

Department of Chemistry

Imperial College London

South Kensington campus

London SW7 2AZ

Tel.: +442075945841

Fax: + 442075945841

E-mail: a.c.spivey@imperial.ac.uk

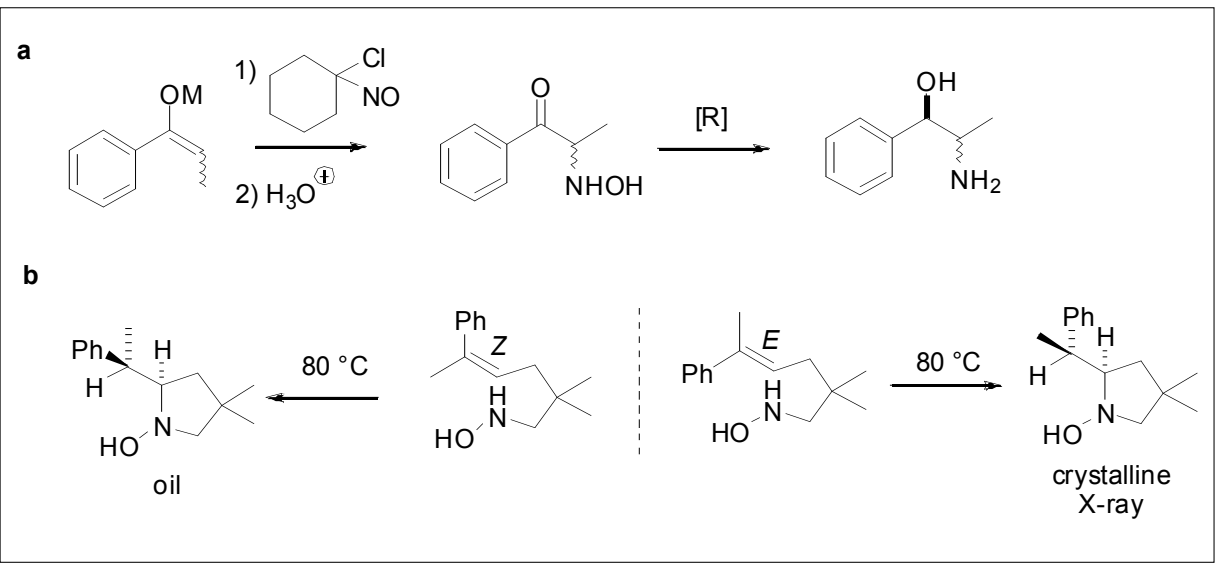

Scheme 1. a) enolate hydroxyamination for potential access to 1,2-aminoalcohols, b) proof of suprafaciality for the retro-Cope elimination.

He then explained that he had to leave immediately as he needed to depart for a skiing week end and that one of his group would explain where I would be staying and which lab I would be working in and directed me down the long corridor of the Science II building before rushing off to his car in the basement. Fortunately, one of his group was still in the labs, Jörg Deerberg, who showed me to 'Residence Oppolzer' - as some flats on Boulevard Carl-Vogt, were colloquially known. That weekend, I met up again with Jörg, who proved to be an immensely helpful and capable $\mathrm{PhD}$ student in our lab, and cleaned and set up my bench, cleared an area of one of the fume-hoods and set going my first experiments - I was confident that this would impress Prof. Oppolzer. It didn't. First thing on Monday morning, a tanned and relaxedlooking Wolfgang Oppolzer strode into my lab and asked how things were going - I explained that I had carried out a couple of reactions in parallel, worked them up and that Jörg had obtained ${ }^{1} \mathrm{H}$ NMRs, but that I couldn't establish the d.e. of either reaction. He appeared deeply unimpressed, proceeded to tell me that I should find another (unspecified) technique to analyse the products and reprimanded me in no uncertain terms for not having fully documented both reactions in my notebook yet, and specifically for not having checked the melting point of one commercial starting material I had used! I realised that the reputation that preceded him was not inaccurate! As indicated above, I had heard many stories of Prof. (von) Oppolzer (also referred to as 'The Baron'), including several which I had previously dismissed as exaggerated relating to his obsession with melting points/mixed melting points, the distillation of sub-microlitre quantities of product, recording of optical rotations at five different wavelengths and many more besides. Looking back, the value that Professor Oppolzer placed on experimental rigour provided invaluable scientific training - at the time it certainly kept his group on its toes! All three of my lab-mates: Jörg Deerberg, Dr. Gajendran Sundarababu ('Babu') and Christian Bochet in addition to being very welcoming and friendly were highly skilled chemists and moreover were adept at handling the somewhat unpredictable visitations to the lab of Prof. Oppolzer; I particularly admired the miraculous change in mood that Babu was able to elicit from a rather perturbed Wolfgang Oppolzer one morning by producing some beautiful 
deep blue crystals of the chloronitrosocamphor reagent he was developing as an enantioselective hydroxyaminating agent.[1] Although he had obtained the crystals several days earlier, Babu's experience and cunning led him to 'reserve' these gems for disclosure to Wolfgang to avert a moment of potential crisis - and the result was a case-study in supervisor management! I think Prof. Oppolzer's obsession with accuracy, scalability and reproducibility (refreshingly, yields for publication were the strict average of several runs not those of a 'one-off, best ever' reaction) for all reactions developed in his laboratories was borne from his time working in the Sandoz company in Basel - another rather splendid legacy of which was that this company underwrote the group's (not inconsiderable) consumables bills on a rolling basis. The Oppolzer labs were extremely well equipped compared to the labs I knew from my PhD with Prof. Sir Jack Baldwin in Oxford.

Professor Oppolzer continued to check on my progress every morning for my first 2-3 weeks - fortunately, I was able to rapidly develop a TFA-derivatisation/GC method for the direct analysis of the d.e.s of my reactions and this greatly pleased him; less fortunately, over the next few months I was unable to optimise the d.e. to a synthetically acceptable level so the project was canned before Easter. This was a great lesson for me - I had observed several projects in Oxford languish due to lack of inspiration or direction so as to drain the confidence and motivation of the researcher; Prof. Oppolzer was decisive; if the objectives could not be obtained in a timely fashion then a project would be dropped and another one explored forthwith. This was not of course universally popular in the group which at that time numbered about 20 , the majority of whom were post-docs (Fig. 1).

Following a short time exploring the hydroxyamination of $\beta$-hydroxyester enolates and then the use of nitroprusside salts as potential electrophilic amination reagents, neither of which yielded satisfactory outcomes, I began work on a project to elucidate the mechanism of a thermal intramolecular ene-type reaction of $\gamma$-alkenyl primary hydroxylamines to form $N$-hydroxypyrrolidines known as the retro-Cope elimination reaction (a hydroamination process in current parlance). My brief for this project was broader than for the previous ones - I was to provide definitive evidence of the stereoselectivity of this type of reaction and then apply the reaction to 'an interesting target molecule'. Prof. Oppolzer expected the reaction to be stereospecific (suprafacial) and proof that this was the case was achieved by preparing a pair of diastereomeric trisubstituted

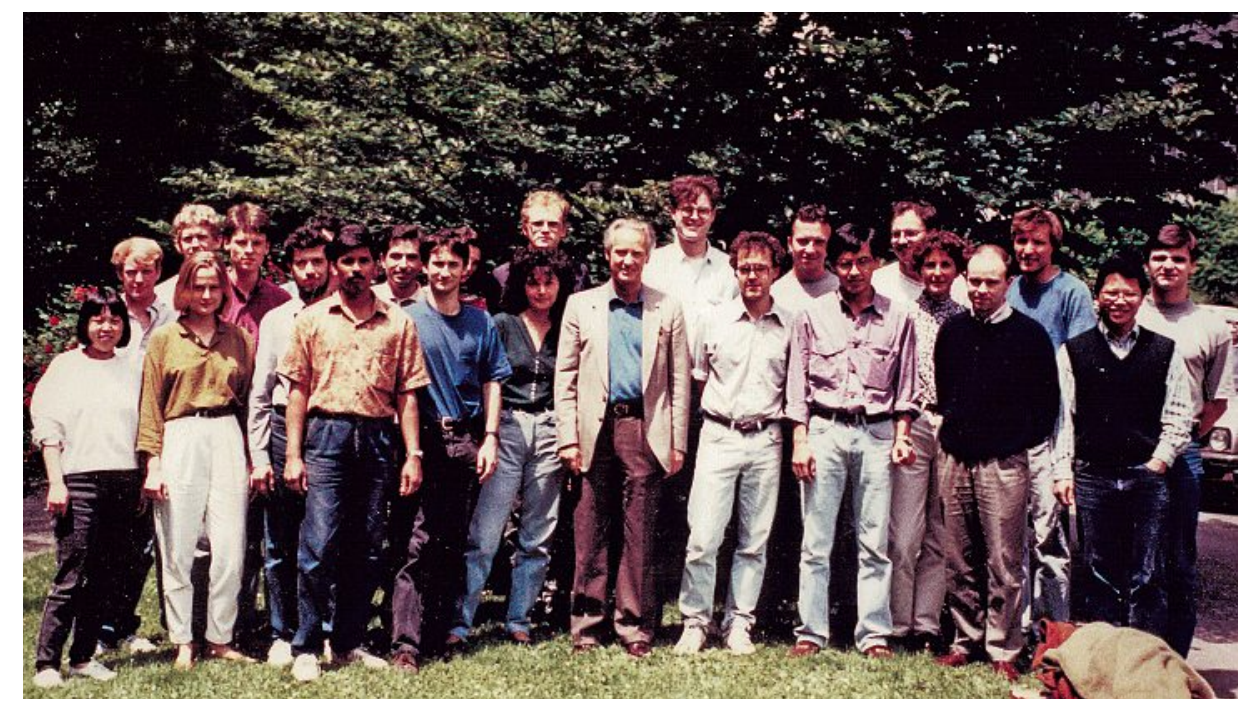

Fig. 1. The Oppolzer Group ca. 1993 (with a few interlopers from other groups on level 2 of Science II!): L to R - Shu Juan Jindra, Jeff Corbett, Chantal Robyr, Alan Spivey, Jörg Deerberg, Boris Seletsky, Jean-Eric Ancel (obscured), Gajendran Sundarababu, Roumen Radinov, Stephane Rosset, Blanda Stammen (largely obscured), Innes Rodriguez, Eric Walther, Wolfgang Oppolzer, Roger Leger, Mike Hutzinger, Imber Flores-Montes, Patrick Lelandais, Bernadette Bourdin, Philipe Liénard, Marcel Signer, Jian Bo Wang, Krysztof Sienkiewicz.

alkenyl substrates, showing that they cyclised exclusively to provide diastereomeric products without crossover and supplying a crystal of one of these products to Dr. Gerald Bernardinelli who performed a single crystal X-ray structure determination to reveal the relative stereochemistry. Designing a pair of substrates that would cyclise efficiently via this rather pleasing reaction had not been entirely straightforward as trisubstituted alkenes were generally very sluggish to cyclise and indeed the gem-dimethyl group present in the tether between alkene and hydroxylamine in the aforementioned substrates was crucial for cyclisation in this case - presumably by virtue of a Thorpe-Ingold effect (Scheme 1b). Exploitation of this reaction in natural product synthesis therefore required careful planning and I spent several long evenings in the library working through the Merck Index and the Encyclopaedia of Natural Products searching for a suitable target on which to exemplify the reaction. Eventually, I settled upon a member of the Lycorine sub-family of the Amaryllidaceae family of alkaloids - this class of target was particularly attractive because previous synthetic work had established that the stereochemistry that I envisioned to be set by the key retro-Cope elimination reaction was 'non-thermodynamic' and difficult to install by several alternative synthetic methods, and also because a simple 'model' system called lycorane, comprising just the basic pentacyclic Galanthan skeleton of this natural product family, was well characterised. With a series of possible synthetic approaches to both the racemic model and actual target alkaloid (+)-trian- thine, mapped out in my mind and backed up with as much literature precedent as I could lay my hands on, I made an appointment to see Prof. Oppolzer to discuss my ideas. Despite my significant trepidation he was inspirationally enthusiastic about the proposal; I was particularly impressed when, as soon as I mentioned the potential targets, he spontaneously sketched the structure of lycorine, complete with all relative strereochemistry on the blackboard on his office. Moreover, and to my great surprise, he was greatly enthusiastic about my suggestion to employ a Pseudomonas Putida microbial oxidation of chlorobenzene to install the initial stereocentres in the $\mathrm{C}$-ring of (+)-trianthine and resolved to procure a sample of the required material from Dr. Gregg Whited, at Genencor in San Franscisco should the model system succeed. Partly at least, I think his enthusiasm to utilize this chemistry stemmed from the fact that much of the pioneering work in this area had been carried out by a former postdoctoral collaborator of his, Tomas Hudlicky. ${ }^{[2]}$ In the event, synthesis of $\alpha$ lycorane proceeded remarkably smoothly: the synthesis of the key precursor was facilitated by prior literature procedures, the retro-Cope elimination proceeded like a dream, and the final steps were well precedented - I obtained more than a gram of the pure product which was very satisfying. As promised, Wolfgang Oppolzer had secured a gift of $500 \mathrm{~g} \mathrm{(!)} \mathrm{of} \mathrm{enantiomerically} \mathrm{pure}$ cyclohexadiene $c i s$-diol and so I next set to work on (+)-trianthine. This proved more challenging and time was tight for its completion before I was scheduled to start a second post-doctoral appointment with 
By the way, Christian Bochet has worked out the transformations $1 \rightarrow 2 \rightarrow 3$. [The cyclization 2 $\rightarrow 3$ is slower $(70 \mathrm{~h}$ in refluxing benzene but similarly efficient : $93 \%$ ! than with your stereoisomer]. I will fax you the revised manuscript (for JACS) within the next weeks for correction.

Back in August, we have also embarked on related routes to (+)-clivonine (which parallel the suggestions of your letter of $27 / 9 / 93$ ) but this project will be finished later.

You will hear from me soon.

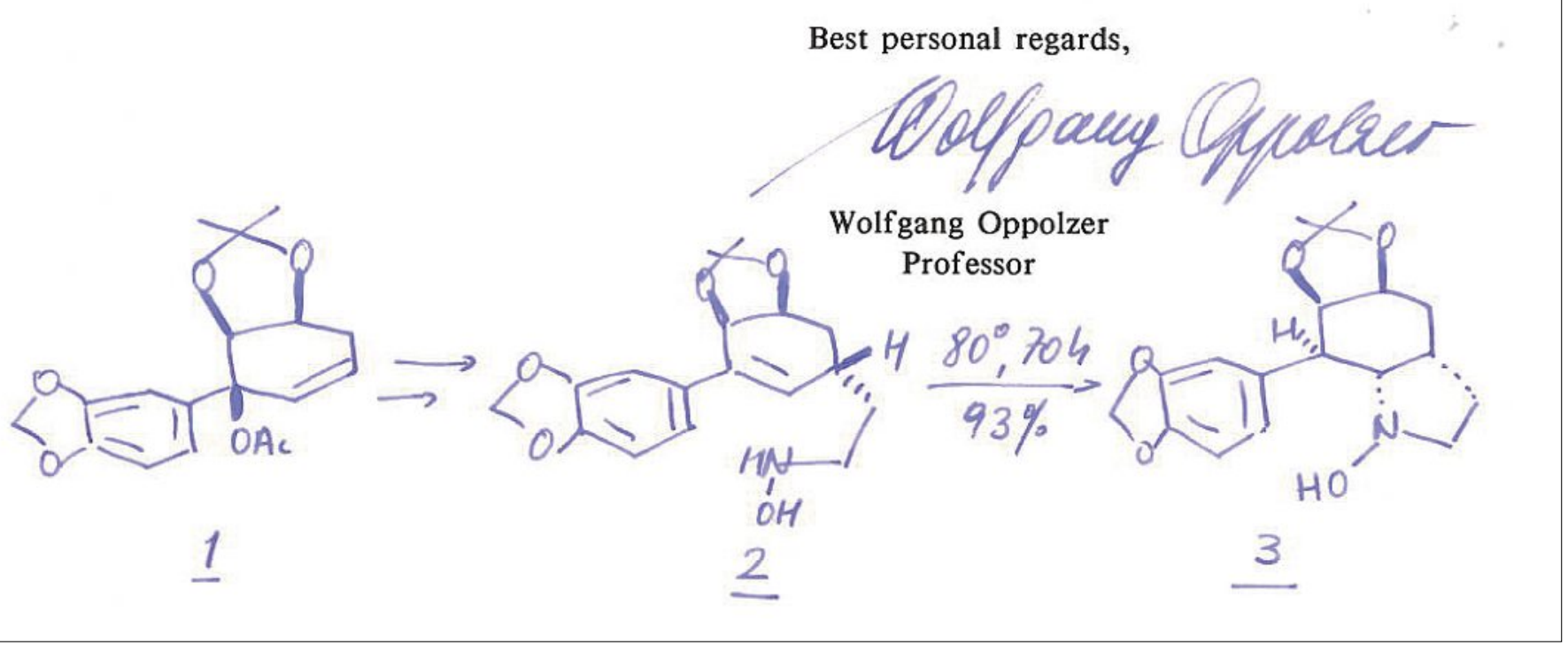

Fig. 2. Excerpt of a letter from Wolfgang Oppolzer to Alan Spivey (dated 3rd Dec 1993) relating Christian Bochet's success at the retro-Cope elimination en route to $(+)$-trianthine. ${ }^{[3]}$

Prof. Sir Alan Battersby in Cambridge. Despite my best efforts, I was unable to complete this synthesis. An allylic substitution reaction gave a mixture of diastereomers, the major epimer of which I carried through a retro-Cope elimination to a compound I thought was (+)-trianthine but for which spectroscopic data (and mp) did not match that reported for the natural product.

I was immeasurably pleased when, on the day I left Geneva, Wolfgang Oppolzer temporarily diverted Christian Bochet from his doctoral studies on the synthesis of Solenopsin and Coniine, to sort out the synthesis of (+)-trianthine for publication. He applied significant pressure on Christian, who over a period of around a month worked round-the-clock to firstly demonstrate that I had prepared the incorrect epimer and thence to develop a highly efficient and fully stereoselective cuprate allylic substitution reaction which allowed him to prepare the natural epimer of (+)-trianthine via a highly efficient retroCope elimination reaction. Wolfgang Oppolzer kept in close contact with me about the progress of the project and was justifiably very impressed with the highly expedient manner in which Christian was able to bring the project to such a satisfactory conclusion (see Fig. 2). The work was published in J. Am. Chem. Soc. early in 1994. ${ }^{[4]}$

In conclusion, my time in Geneva was in immensely formative time for me from a scientific perspective. It was also a great time from a personal perspective; Blanda Stammen, now my wife, was also a post doc in the Oppolzer Group! Although Wolfgang Oppolzer demanded productivity in his laboratories he was not prescriptive about working hours; indeed, so long as you were fully focused on the chemistry during the week he considered the weekend squandered - at least during the skiing season - if you did not take advantage of the proximity of Geneva to some of the best piste in Europe. In my case, I was a novice skier so spent more time cycling than skiing but the work hard, play hard ethos he epitomized characterized my 18 months in Geneva.

Received: October 10, 2009

[1] W. Oppolzer, O. Tamura, G. Sundarababu, M. Signer, J. Am. Chem. Soc. 1992, 114, 5900.

[2] T. Hudlicky, J. W. Reed, Synlett 2009, 685.
[3] The synthesis of clivonine that Wolfgang Oppolzer refers to was started by Christian Bochet and further work carried out subsequently by Hervé DuMartin, Helmut Haning and Laure Guy in Geneva but never completed or any part published. We have recently completed this synthesis which will hopefully be published shortly.

[4] W. Oppolzer, A. C. Spivey, C. G. Bochet, J. Am. Chem. Soc. 1994, 116, 3139. 